erstattung wahrgenommen wird. Als Ergebnis kann die so genannte „Vorzugslesart“ (S. 226) von Frauen festgehalten werden, „weil sich diese [...] gegen Widerstände von unten nach oben gekämpft haben und deshalb primär nicht für die Ausübung von Macht stehen“ (ebd.). Macht sei Gebiet des Mannes, womit die in den wissenschaftlichen Analysen ausgemachte „Norm“ bestätigt werde. Das Vorhandensein weiblicher Führungskräfte in diesem Gebiet finde zwar Respekt und Zustimmung, ihr Erfolg werde aber vorrangig über die Aneignung männlich konnotierter Eigenschaften erklärt. Die Frau bleibe somit „Exotin“. Darüber hinaus werde der Erfolg individualisiert und folglich strukturelle Ursachen sozialer Ungleichheit ausgeblendet, obwohl letztere gleichzeitig innerhalb der geführten Gruppendiskussionen erkannt wurde.

Das Buch „Ungleich mächtig“ gibt einen umfassenden Einblick in die Funktionsweise der deutschen Medienlandschaft in Hinblick auf die Konstruktion von Geschlecht. Wesentliche Erkenntnisse der Studien zirkulierten zwar vorher bereits als Vermutungen in der öffentlichen Diskussion über die weibliche Unterrepräsentanz, werden nun aber wissenschaftlich fundiert. Gerade die Berücksichtigung der verschiedenen Blickwinkel in der Analyse führt somit zu einer umfangreichen Bestandsaufnahme.
$\mathrm{Zu}$ kritisieren bleibt, dass der eingangs formulierte Anspruch, „erstmalig [...] mit den Bereichen Politik, Wirtschaft und Wissenschaft drei gesellschaftliche Felder und deren unterschiedliche Repräsentationen vergleichend in den Blick [zu nehmen]“ (S. 7), nicht erfüllt werden kann. Stets fallen vor allem Wissenschaft, aber auch Wirtschaft, mangels zu erhebender Daten, im Vergleich hinter die Analyse der Politik zurück. Unberücksichtigt bleibt auch der Einbezug neuer Medienformate (Blogs, Mags, Social Media-Anwendungen, Kommentarfunktionen etc.) durch das Internet, die eher ein Aufbrechen medial konstruierter Geschlechterverhältnisse vermuten lassen als die analysierten klassischen Medien.

Dies schmälert letztlich aber nicht die durch die Lektüre des Buches gewonnenen Einsichten. Gerade für die Arbeit des djb im Rahmen des Projekts „Aktionärinnen fordern Gleichberechtigung“ ist es von großem Interesse, sein Anliegen - die Erhöhung des Frauenanteils in Führungspositionen in der Wirtschaft - in einen gesamtgesellschaftlichen Kontext zu bringen. Mit klaren Statements kann so der permanenten Verortung in der „Frauensparte“ etwas entgegengesetzt werden, wovon sich im Idealfall auch die anderen 50 Prozent der Gesellschaft angesprochen fühlen.

\title{
Das Deutsche Anwaltsinstitut e. V. sucht Referentinnen
}

Ungleich zu vielen anderen Berufen sind Frauen in der Anwaltschaft heute keineswegs unterrepräsentiert. Der Frauenanteil ist hier während der letzten 40 Jahre von 4,5 Prozent auf 32 Prozent gestiegen. Auch in Zukunft geht der Aufwärtstrend erst einmal im gleichen Tempo weiter. Bereits im Wintersemester 2004/2005 waren mehr als 50 Prozent der JuraStudierenden Frauen. Inzwischen bewegt sich deren Anteil unaufhaltsam auf die 60-Prozent-Marke zu.

Diese Tendenz findet sich auch in den Teilnehmerzahlen des Deutschen Anwaltsinstituts e. V. (DAI) wieder, der Ausund Fortbildungseinrichtung von Bundesrechtsanwaltskammer, Bundesnotarkammer und 43 Rechtsanwaltskammern und Notarkammern und eines der bundesweit führenden Fortbildungsinstitute für Rechtsanwälte, Fachanwälte, Notare und deren Nachwuchs sowie Mitarbeiter. Bei den unter 35-Jährigen sind es über 50 Prozent Frauen, die an den Seminaren, Fachlehrgängen und Arbeitstagungen des Deutschen Anwaltsinstituts teilnehmen. Diese Zahl schließt sowohl die eigenen Aus- und Fortbildungsstätten in Bochum, Berlin und Heusenstamm bei Frankfurt am Main ein als auch Teilnehmerstatistiken aus mehr als 50 Orten in Deutschland, in denen das Deutsche Anwaltsinstitut seine insgesamt 700 Veranstaltungen mit rund 30.000 Teilnehmerinnen und Teilnehmern jährlich durchführt.

Diesem erfreulichen Aspekt bei der Teilnehmerentwicklung steht jedoch leider eine gähnende Leere in den Rängen von
Referentinnen gegenüber. Denn wie eine Überprüfung des DAI ergeben hat, mangelt es in lehrenden Institutionen einschließlich der juristischen Fakultäten der Hochschulen nach wie vor an Referentinnen und Dozentinnen. Der Anteil der Referentinnen in Aus- und Fortbildungsinstitutionen liegt lediglich bei rund 11 Prozent, in den juristischen Fakultäten der Universitäten bei knapp 16 Prozent, Tendenz sinkend.

Das DAI möchte daher das zweifellos vorhandene, aber bisher weitgehend ungenutzte Potential von Referentinnen für seine Veranstaltungen mit einer bundesweiten Initiative aktivieren und dazu motivieren, erworbene juristische Kenntnisse und Erfahrungen in einem seriösen und effektiven Rahmen an die nächste Generation von Rechtsanwälten und Notaren weiterzugeben. Gesucht werden Anwältinnen, Notarinnen, Richterinnen und Professorinnen, die als Referentinnen im DAIFortbildungsprogramm neue Impulse setzen wollen.

\section{Bitte kontaktieren Sie:}

Dr. Katja Mihm

Geschäftsführerin

Rechtsanwältin, Fachanwältin für Arbeitsrecht, Mediatorin

Deutsches Anwaltsinstitut e. V.

Universitätsstr. 140

44799 Bochum

Tel.: 02349706411

E-Mail: mihm@anwaltsinstitut.de 